\title{
Evaluación de acciones explosivas sobre estructuras de hormigón armado mediante elementos finitos
}

\section{Evaluation of Blast Loads on Reinforced Concrete Structures with Finite Elements}

$\underline{\text { M. Bermejo }}^{(*)}$, A. P. Santos ${ }^{(*)}$, J. M. Goicolea ${ }^{(* *)}$, A. Pérez ${ }^{(*)}$

RESUMEN

El fenómeno de explosión sobre estructuras de hormigón tiene efectos en muchos casos catastróficos a pesar de su reducida frecuencia. Las edificaciones civiles no suelen estar diseñadas para resistir este tipo de solicitación dinámica, por lo que conviene disponer de una metodología que permita analizar los efectos de las explosiones sobre las mismas. Este trabajo estudia el comportamiento de las estructuras reticuladas de hormigón armado frente a estas acciones, mediante métodos numéricos de elementos finitos lagrangianos con integración explícita en el tiempo. Se analizan de forma realista partes de la estructura como columnas y forjados usando modelos con hormigón y armaduras de forma segregada, pero las limitaciones computacionales los hacen inviables para estructuras completas. Se proponen modelos de elementos lámina y viga debidamente calibrados para obtener una respuesta similar.

Se obtienen conclusiones para el uso y calibración de modelos y simulaciones realistas de edificios completos para estudios de seguridad.

Palabras clave: Explosión; estructura reticulada hormigón; losa reticulada; MEF explícitos; láminas.

\section{ABSTRACT}

Explosive phenomena on concrete structures have catastrophic effects in many instances despite its reduced occurrence. The civil buildings are usually not designed to withstand this type of dynamic load, so a methodology to analyze the structural response on blast loads is recommended. This paper studies the behavior of reinforced concrete frame structures against these actions by lagrangian finite elements method with explicit time integration. Finite element models of segregated concrete and rebar are used to make possible the study of structure parts as columns and slabs, but is not possible to use these detailed models in complete structures because of excessive computational costs. Shell and beam elements models properly calibrated are needed to obtain a similar response.

Conclusions and practical recommendations are provided for the use and calibration of models and realistic simulations.

Keywords: Blast; RC frame-structure; waffle slab; explicit FEM; shells.

(*) ETSI Minas - Universidad Politécnica de Madrid, (España).

(**) ETSI Caminos, Canales y Puertos - Universidad Politécnica de Madrid, (España).

Persona de contacto/Corresponding author: mario.bermejo@upm.es (M. Bermejo).

Cómo citar este artículo/Citation: Bermejo, M., Santos, A. P., Goicolea, J. M., Pérez, A. (2015). Evaluación de acciones explosivas sobre estructuras de hormigón armado mediante elementos finitos. Informes de la Construcción, 67(539): eo95, doi: http://dx.doi. org/10.3989/ic.13.121.

Licencia / License: Salvo indicación contraria, todos los contenidos de la edición electrónica de Informes de la Construcción se distribuyen bajo una licencia de uso y distribución Creative Commons Reconocimiento no Comercial 3.o. España (cc-by-nc). 


\section{INTRODUCCIÓN}

En los últimos años varios edificios civiles en todo el mundo (1) han sido objeto de ataques terroristas por medio de cargas explosivas. En el diseño de estos edificios, mayoritariamente construidos en hormigón armado, no se consideró la posibilidad de acciones explosivas o de impacto, que si bien tienen poca probabilidad de ocurrir, son capaces de causar efectos catastróficos.

En España han sucedido atentados como el de la Terminal 4 de Barajas el 30 de diciembre de 2006, que con una carga de entre 500 y $800 \mathrm{~kg}$ de explosivo produjo 2 muertos y 52 heridos y el colapso de la mayor parte de las cinco plantas de uno los módulos del aparcamiento (2), o el de Burgos contra un edificio de la Guardia Civil el 29 de julio de 2009, ocasionando 69 heridos y graves daños al edificio con una furgoneta con más de $300 \mathrm{~kg}$ de explosivos. Esto muestra la necesidad de contemplar en el diseño de estructuras civiles las posibles acciones de explosiones e impactos.

Históricamente los estudios de acciones explosivas mediante elementos finitos con integración explícita comienzan con el objetivo de simular el efecto de armas nucleares. El código HEMP (3) (1964) de M.Wilkins permitió el análisis en dos dimensiones y en 1975 surge HEMP3D (4). En 1978 J.O.Hallquist desarrolló en el Lawrence Livermore National Laboratory la primera versión de DYNA3D (5) que incluye elementos finitos estructurales y que en los años siguientes continúa su desarrollo para otras aplicaciones. En 1988, a partir de los desarrollos anteriores, J.O.Hallquist y D.J.Benson crearon LS-DYNA (6).

El modelo de hormigón que se ha empleado en este trabajo surgió de la implementación de Pelessone (7) de un modelo de plasticidad con cierre a compresión (cap). Entre 1994 y 1997 Schwer y Murray (8), basándose en los trabajos de Simó y Ju (9), incluyeron una formulación viscoelástica para tener en cuenta los efectos por velocidad de deformación y varias formas de daño que hacen posible modelar el ablandamiento y fallo del hormigón.

Se han realizado diversos estudios en los últimos años, varios de ellos referentes a la respuesta de elementos o estructuras de hormigón armado. En cuanto a la investigación sobre elementos estructurales tales como vigas y losas se puede citar el trabajo de Magnusson (10) (2007) sobre la respuesta dinámica de vigas de hormigón armado, usando métodos basados en reducción a modelos de un grado de libertad para analizar la respuesta y su posterior utilización en el diseño de estructuras. También el estudio de Silva (11) (2009) en el que se evalúa el comportamiento de losas de hormigón con diferentes cuantías de armado y diferentes cargas explosivas, analizando la respuesta mediante el método displacement based design. En esta misma línea, con el objetivo de estudiar cargas explosivas sobre losas y validar los modelos de hormigón implementados en el código LS-DYNA (6), Morales-Alonso et al (12) (2011) desarrollaron un trabajo de ensayos y simulación sobre losas armadas de $50 \times 50 \mathrm{~cm}$.

En cuanto a la evaluación de edificios completos Luccioni et al (13) (14) (2003, 2004) estudiaron el atentado contra la Asociación Mutual Israelita Argentina en 1994 mediante la utilización de análisis dinámico explícito con interacción fluido-estructura en el código AUTODYN (15), con un mode- lo homogeniezado estimando daños a la estructura y colapso progresivo. Krauthammer (16) (2007) estudió el colapso progresivo en un edificio completo usando el código ABAQUS/ Explicit (17). Jayasooriya et al (18) (2011) proponen un método para analizar elementos de un edificio cuyo fallo puede llevar al colapso.

Paralelamente se han desarrollado nuevos modelos de material de hormigón para su empleo en el cálculo por elementos finitos y técnicas para computar correctamente la armadura embebida de forma segregada. Así, en el análisis de la respuesta de una barrera de hormigón armado de puente de ferrocarril ante el impacto de un bogie ferroviario, Akram (19) (2008) utiliza LS-DYNA para modelar hormigón y barras de acero de forma segregada, restringiendo el movimiento de los elementos de acero en la malla de hormigón, lo que permite calcular la respuesta común del conjunto hormigónarmadura.

Las recientes mejoras en la capacidad de computación, en los modelos de material y en las técnicas de modelado de los códigos de elementos finitos permiten una mejor aproximación al problema de evaluar la acción explosiva sobre estructuras. Así, este trabajo presenta una estrategia de modelado para edificios reticulares de hormigón armado, utilizando la potencia de los últimos modelos de material y formulaciones implementadas en el código LS-DYNA. Dicha estrategia parte de la validación de modelos detallados de elementos de continuo 3D, que permiten calibrar modelos simplificados de elementos viga y lámina, con los que es posible analizar con mayor precisión que hasta el momento los efectos de cargas explosivas sobre edificios de dimensiones del orden de las decenas de metros. Permite además comparar distintas propuestas de mejoras de diseño con el objeto de evitar o reducir, en la medida de lo posible, los daños y el posible colapso posterior de la estructura.

En el resto de este artículo, en el apartado 2 se discuten los modelos de material y explosión así como las distintas técnicas de modelado empleadas; en el apartado 3 se describen y validan los modelos de elementos de continuo; en el apartado 4 se valida la metodología empleada para obtener modelos de elementos estructurales realistas; y en el apartado 5 se proponen aplicaciones tales como el cálculo de un pórtico y un edificio completo sometidos a acción explosiva. En el apartado 6 se recogen las observaciones finales, recomendando el empleo de modelos con elementos estructurales adecuadamente calibrados para el estudio de las explosiones.

\section{ELEMENTOS DE LOS MODELOS}

Los códigos de simulación numérica para cálculo dinámico permiten, mediante el uso de métodos de elementos finitos lagrangianos con integración explícita en el tiempo, el estudio de la acción explosiva en los tiempos muy cortos en los que actúa (20). En este trabajo se emplea el código LS-DYNA (6) para simular explosiones sobre estructuras reticuladas de hormigón, utilizando un modelo de material recientemente implementado en dicho código para hormigón, el CSCM (Continuous Surface Cap Model) (21) para elementos de continuo 3D, junto con la técnica CONSTRAINED_LAGRANGE_IN_SOLID que proporciona la debida compatibilidad con las armaduras, consideradas individualmente mediante elementos viga. Este procedimiento permite estudiar de forma realista modelos detallados de columnas y forjados, usan- 
do geometrías muy cercanas a las reales y modelos no lineales de los materiales.

Sin embargo, con la potencia de cálculo disponible en la actualidad no es posible, debido al alto coste computacional, emplear estos modelos detallados de elementos de continuo $3 \mathrm{D}$ en estructuras con dimensiones de varias plantas. Este coste en los modelos depende principalmente del número de elementos y del tamaño mínimo del elemento empleado; en estos casos es necesaria una cantidad elevada de elementos de pequeño tamaño para conseguir una geometría realista y pasos de tiempo muy reducidos, haciendo inviable el cálculo.

Como alternativa se ha desarrollado un método para utilizar modelos de elementos estructurales, elementos viga y lámina, con un adecuado ajuste de parámetros y propiedades. En este caso se usa el modelo de material CONCRETE_EC2 de LS-DYNA, diseñado para la simulación de hormigón armado con este tipo de elementos, y un procedimiento para incluir la armadura segregada de forma adecuada. Mediante este modelado con elementos estructurales es posible reproducir con suficiente aproximación el comportamiento de modelos detallados realistas de forjados y columnas de estructuras reticuladas de hormigón frente a acciones explosivas, posibilitando el análisis de una estructura de varias plantas frente a explosión. En el apartado 2.2 se explican someramente los modelos de hormigón.

El modelado requiere de varios componentes para lograr obtener una respuesta realista. A continuación se describen brevemente las características y problemáticas de cada uno de ellos.

\subsection{Cálculo explícito de elementos finitos}

El método de elementos finitos permite obtener una solución numérica aproximada sobre un medio continuo en el que están definidas las ecuaciones del movimiento que caracterizan el comportamiento físico del problema, mediante una formulación variacional o débil para las ecuaciones del movimiento y la construcción de una solución aproximada basada en coordenadas nodales y funciones de forma de elementos (22). En el caso de los métodos de elementos finitos explícitos se emplea un algoritmo de integración explícita en el tiempo, siendo el método de diferencias centrales el utilizado en LSDYNA.

Este procedimiento logra el desacoplamiento de las ecuaciones globales del movimiento de forma que éstas se plantean y resuelven de forma local, elemento por elemento. El método es válido cuando el intervalo de integración $\Delta t$ es tan corto que nodos contiguos de la malla no tienen tiempo de comunicarse entre sí durante ese mismo intervalo. Esta limitación se concreta en la condición de Courant $\Delta t \leq \Delta t_{\text {crit }}$ que debe mantenerse para una integración estable, siendo $\Delta t_{\text {crit }}$ el tiempo que tarda la onda de tensión más rápida en recorrer el espacio que separa los nodos. En el caso de que no se cumpla la condición de Courant el método se hace automáticamente inestable, produciendo energía no física en el cálculo.

El tamaño del elemento es clave, puesto que cuanto más fina es la malla el $\Delta t$ es menor, aumentando el coste computacional no sólo por el número de grados de libertad del modelo, sino también por el número de pasos de tiempo.
En los métodos explícitos todas las operaciones se realizan elemento por elemento a nivel local y no se necesita ensamblar ninguna matriz de coeficientes global, ni resolver sistemas de ecuaciones simultáneas. Como consecuencia, la necesidad de memoria crece de manera lineal con el tamaño del problema, en lugar de cuadrática como en los métodos implícitos, lo que posibilita la solución eficaz de grandes problemas tridimensionales.

Por todo ello la elección del método se ajusta al problema, ya que en casos de dinámica rápida en grandes simulaciones $3 \mathrm{D}$ los modelos explícitos son más eficientes computacionalmente y más robustos para cálculo no lineal.

\subsection{Modelos de hormigón}

El hormigón es un material que en las condiciones extremas que nos ocupan, en las que se alcanza la resistencia última, tiene un comportamiento complejo, no lineal. Presenta una resistencia mucho menor a tracción que a compresión, fisuración en tracción, contribución del hormigón entre fisuras (tension stiffening), plastificación en compresión, aumento de características resistentes cuando está sometido a altas velocidades de deformación, así como límites de rotura diferentes dependiendo de la forma de fallo (tracción, compresión, cortante). Otros fenómenos como la retracción y la fatiga no intervienen en este caso.

El modelo de material usado para los elementos de continuo 3D es el CONCRETE_CSCM implementado en LS-DYNA. Este material, que sólo esta disponible para elementos de continuo y no para elementos viga o lámina, tiene las siguientes características (Figura 1a):

- Diferente comportamiento a tracción y a compresión.

- Tres superficies de fallo:

- Fallo dominado por cortante.

- Fallo dominado por compresión.

- Fallo dominado por tracción.

- Endurecimiento y ablandamiento por daño dúctil, en compresión.

- Comportamiento cuasi-frágil con daño y rotura en tracción debido a la fisuración.

· Erosión: eliminación de elementos con una deformación dada una vez alcanzado un límite de daño. Los elementos son eliminados del cálculo cuando la variable de daño isótropa es mayor de 0,99 y la deformación máxima principal llega al límite establecido por $\xi_{\text {erode }}$.

El modelo se puede definir a partir de 4 parámetros: densidad $(\rho)$, límite elástico en compresión no confinada $\left(f^{\prime} c\right)$, tamaño máximo de árido $\left(D_{\text {agg }}\right)$ y una condición de erosión $\left(\xi_{\text {erode }}\right)$, siendo generados el resto de parámetros de forma automática por el propio código para un hormigón estructural estándar. No obstante si se desea una definición detallada se dispone de un total de 37 parámetros posibles, cuyas descripciones y rangos vienen recogidos en el manual de LSDYNA para el material hormigón 159 (21) por lo que no se explicitan aquí.

El modelo de material usado para los elementos estructurales es el modelo de LS-DYNA CONCRETE_EC2 (23), que puede ser usado en el modelado de elementos viga y 

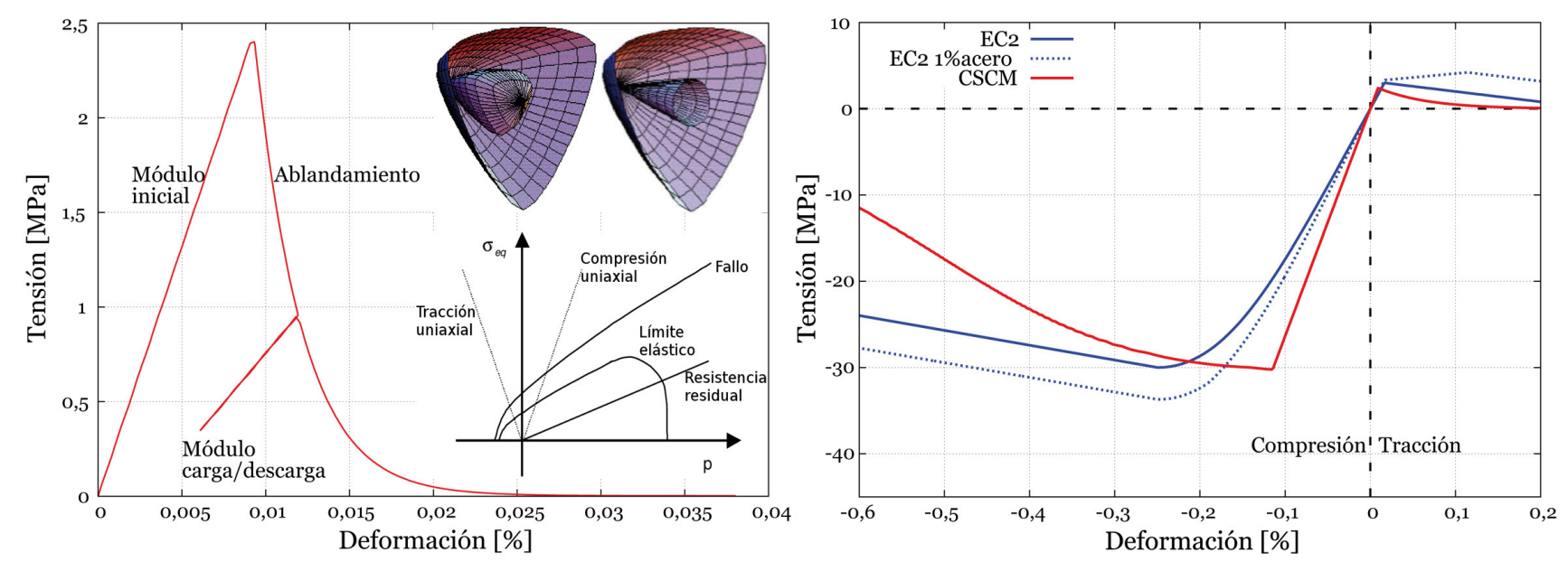

Figura 1. a) Material CSCM para hormigón de $30 \mathrm{MPa}$ en tracción uniaxial no confinada, superficies de fallo 3D y cortes de la superficie en el plano desviador (21). b) Hormigón de $30 \mathrm{MPa}$ en tracción y compresión uniaxial. Curva CSCM para elementos de continuo y material CSCM. Curvas EC2 y EC2 1 \% acero para elementos estructurales y material EC2, sin cuantía de acero y con cuantía del $1 \%$.

lámina pero no en elementos de continuo $3 \mathrm{D}$, a diferencia del CSCM. La Figura 1b muestra el diagrama tensión-deformación de un hormigón de $30 \mathrm{MPa}$ modelado con este material y lo compara con el material CSCM. Este material tiene gran parte de las propiedades exigidas anteriormente: diferente comportamiento a tracción y a compresión, con ablandamiendo en compresión, con daño debido a fisuración en tracción y con la capacidad de erosionar para una deformación dada gracias a una formulación adicional. Además incluye una formulación homogeneizada que permite considerar cuantías de acero en cada elemento. Esta última opción se emplea sólo para considerar el armado transversal, ya que el armado longitudinal se modela de forma segregada.

\subsection{Modelos de armado y su unión con el hormigón}

El acero es un material no lineal con plastificación y rotura tanto a tracción como a compresión y adicionalmente, al igual que en el hormigón, el efecto de aumento del límite elástico con la velocidad de deformación. Para modelar el acero de armado se usan elementos viga o barra con modelo de material PIECEWISE_LINEAR_PLASTICITY (23) implementado en LS-DYNA, que incluye deformación plástica al llegar al límite elástico, aumento de la tensión de plastificación con la velocidad de deformación y rotura.

El hormigón armado se puede simular mediante un modelo homogeneizado, usando un sólo modelo de material que incluye la contribución del armado, o con modelo segregado, empleando dos modelos de material, uno para hormigón y otro para acero, en dos mallas superpuestas. Éste último es el utilizado en este trabajo dado que el homogeneizado, aunque es más sencillo, presenta serias limitaciones en la evaluación detallada de fenómenos de grandes deformaciones, plastificación y rotura de elementos estructurales. Para el modelado segregado de hormigón y acero existen dos opciones: la primera es hacer coincidir los nodos de las mallas de hormigón y acero, de forma que al compartir nodos comunes los materiales se deforman solidariamente y por tanto suman su respuesta frente a solicitaciones; la segunda es usar la formulación CONSTRAINED_LAGRANGE_IN_SOLID de
LS-DYNA (19) (23) que permite situar los elementos viga de acero en cualquier parte de la malla de elementos de continuo de hormigón. Esta formulación permite imponer la compatibilidad de deformaciones entre elementos viga embebidos en los sólidos sin necesitar que los nodos de las vigas sean comunes con los de los elementos sólidos, con una disposición geométrica cualquiera dentro del elemento. Sin embargo esta opción técnica no se puede utilizar en modelos estructurales que utilizan láminas para el hormigón. En este caso deben coincidir los nodos de las dos mallas, aunque se permite introducir una excentricidad de los nodos de las vigas que produce su colaboración plena a flexión. Se realiza mediante el comando BEAM_OFFSET (23) que permite considerar la excentricidad de las armaduras respecto al plano en el que son definidas (24).

La Figura 2 muestra un ejemplo de validación para la formulación de materiales segregados. Tiene unas dimensiones de $1 \times 1 \times 3 \mathrm{~m}$ con tres elementos de hormigón y embebidas en esa malla tres barras de acero de sección $0,005 \mathrm{~m}^{2}$, con materiales hormigón CSCM de $30 \mathrm{MPa}$ y acero de $240 \mathrm{MPa}$. Se trata de un modelo dinámico y explícito en el que la probeta definida es sometida a tracción mediante una fuerza impues-

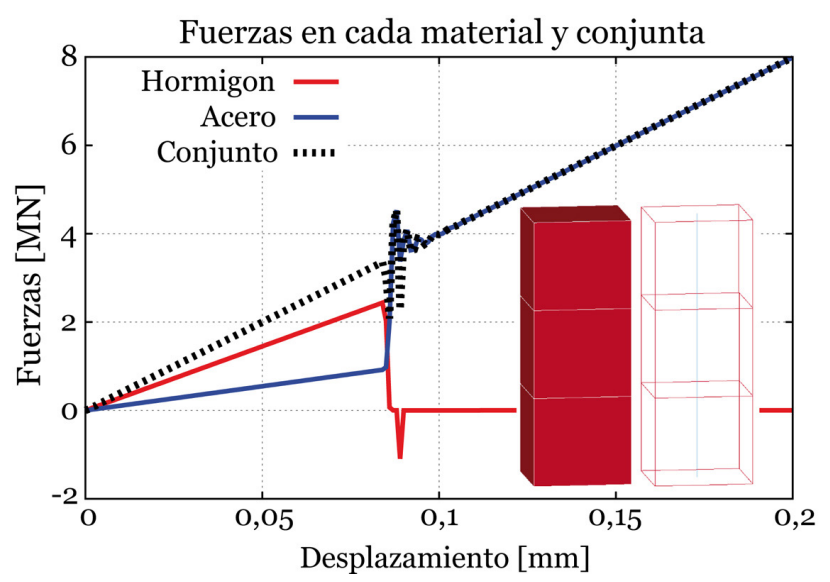

Figura 2. Validación de la formulación CONSTRAINED_LAGRANGE_ IN SOLID: Malla del modelo (hormigón $30 \mathrm{MPa}$, acero $240 \mathrm{MPa}$ ) y gráfica de la evolución de la carga a tracción con fisuración. 
ta aplicada en un extremo de forma lineal. Al alcanzarse la rotura del hormigón a tracción esta fuerza pasa a ser soportada totalmente por el acero con unas oscilaciones intermedias debido a la discontinuidad. En la figura se muestra la fuerza conjunta de hormigón y acero y las fuerzas transmitidas por cada uno de los materiales.

\subsection{Modelos de la acción explosiva}

El fenómeno físico se origina en un material explosivo que varía su estado químico produciendo un elevado incremento de volumen, con la consiguiente variación en la presión. Ésta variación se propaga como una onda de elevada presión en un tiempo muy corto por el medio circundante, que incide sobre la estructura y a su vez produce una onda reflejada que se compone con la anterior. Esta onda de presión reflejada tiene la misma forma que la de la presión incidente pero el pico de presión es mayor, pudiendo alcanzar, dependiendo de la inclinación con que llega al obstáculo y de la potencia de la explosión, hasta doce veces el valor correspondiente a la onda incidente (25).

Existen dos métodos fundamentales para transferir las presiones generadas en la explosión a la estructura: 1) Aplicación de las presiones mediante el empleo de funciones temporales de la presión suministradas por fórmulas empíricas -metodología CONWEP (26)-; 2) Aplicación de las presiones mediante el uso de modelos acoplados y muy detallados de dinámica de fluidos (CFD).

El primer método, que tiene un coste computacional reducido, es adecuado para el estudio de modelos de geometrías sencillas. Calcula todos los efectos geométricos de la llegada de la onda a distintas zonas de la estructura y su reflexión, pero no tiene en cuenta los efectos de las ondas reflejadas sobre otros paramentos ni considera la transmisión de la onda explosiva por las aberturas producidas. Sin embargo la consideración de las ondas reflejadas en un caso de edificio abierto es poco importante. El segundo método tiene un coste computacional elevado, tiene en cuenta los fenómenos de reflexión, la parte de la onda que se transmite entre forjados y es necesario para el análisis de geometrías complejas, pero no es adecuado para el estudio de grandes modelos porque necesitaría recursos de cálculo prohibitivos.

Por ello en este trabajo, con una clara orientación práctica, se ha usado el primer método. Se utiliza el comando LOAD_BLAST_ENHANCED (23) de LS-DYNA que es una mejora de la implementación de Ranhders-Pehrson y Bannister (27) en DYNA3D. Dicha implementación usa las ideas y datos del trabajo de Kingery y Bulmash (28). Proporciona unos valores de presiones coincidentes con el código CONWEP (Conventional Weapons Effects), de acuerdo a los resultados experimentales recogidos en el TM-5-855-1 (25).

\section{MODELOS DE ELEMENTOS FINITOS DE CONTINUO 3D}

\subsection{Características de los modelos de continuo $3 \mathrm{D}$}

La masa de hormigón se representa mediante una malla de elementos finitos de continuo hexaédricos y el armado se modela mediante elementos tipo viga, con la disposición, longitud y diámetro reales. Ambas mallas se unen mediante CONSTRAINED_LAGRANGE_IN_SOLID. Los modelos de material empleados son los reseñados en los apartados anteriores, modelo de material CSCM para el hormigón y modelo de material linear piecewise plasticity para el acero.

\subsection{Validación de respuesta con resultados de ensayos}

Se realiza la comprobación de un modelo de elementos de continuo con un ensayo de explosión sobre losas de hormigón analizado en el artículo de Silva y Lu (11). En estos ensayos se disponen varias losas de hormigón armado y se las somete a distintas cuantías de explosivo a diferentes distancias (Tabla 1). Hay dos tipos de losas a ensayar, ambas apoyadas sobre dos vigas de acero con dimensiones $120 \times 120 \times 9 \mathrm{~cm}$ armadas en una sola cara con diferentes cuantías: la serie (I) con $0,50 \%$ de cuantía y la serie (II) con 0,18 \% de cuantía. Los materiales empleados son hormigón de 27,6 MPa y acero de $414 \mathrm{MPa}$, con módulo elástico de $200 \mathrm{GPa}$ y tensión de rotura de $621 \mathrm{MPa}$.

En el modelado se emplea una malla de elementos finitos de $40 \times 40 \times 3$ elementos de continuo con material CSCM CONCRETE (características: $\rho=2320 \mathrm{~kg} / \mathrm{m}^{3}, f^{\prime} c=27,6 \mathrm{MPa}$, $D_{\text {agg }}=19 \mathrm{~mm}, \xi_{\text {erode }}=1,05$ y una malla de elementos viga de material elastoplástico (características: $\rho=7850 \mathrm{~kg} / \mathrm{m}^{3}$, $\mathrm{E}=200 \mathrm{GPa}, v=0,3, \sigma_{v}=414 \mathrm{MPa}$, deformación de rotura $\left.\varepsilon_{u}=14 \%\right)$ que simulan la armadura.

El armado forma una malla de 1,08 × 1,08 m. En los casos (I) consta de siete barras en cada una de las dos direcciones del plano de la losa, dos en cada extremo y las otras cinco equidistantes. En los casos (II) consta de tres barras equidistantes, también en ambas direcciones. En todos los casos se simulan mediante elementos viga de $8 \mathrm{~cm}$ de longitud.

Calculando estos modelos con la metodología explicada se obtiene una buena correlación entre los daños del ensayo y los calculados por elementos finitos (Figura 3). En los ensayos se observan: para las losas I-a y II-b fisuras menores de un milímetro; en la losas I-c y II-c fisuras mayores de 3 milímetros y desplazamientos residuales de $22 \mathrm{~mm}$ y $16 \mathrm{~mm}$. Estos resultados son consitentes con los modelos, en los que se muestra el mapa de deformación plástica y zonas erosionadas, y en los que se obtienen desplazamientos residuales de $24 \mathrm{~mm}$ para el caso I-c y de $13 \mathrm{~mm}$ para el caso II-c.

Tabla 1. Casos analizados dentro de cada serie (11).

\begin{tabular}{|l|l|l|l|l|l|l|l|l|}
\hline \multicolumn{1}{|c|}{ Caso } & I-a & \multicolumn{1}{c|}{ I-b } & \multicolumn{1}{c|}{ I-c } & \multicolumn{1}{c|}{ I-d } & II-a & II-b & II-c & II-d \\
\hline Carga (kg TNT) & 0,41 & 0,77 & 2,00 & 2,83 & 0,29 & 0,54 & 1,00 & 1,41 \\
\hline Distancia (m) & 0,9 & 0,9 & 0,3 & 0,3 & 0,9 & 0,9 & 0,3 & 0,3 \\
\hline
\end{tabular}




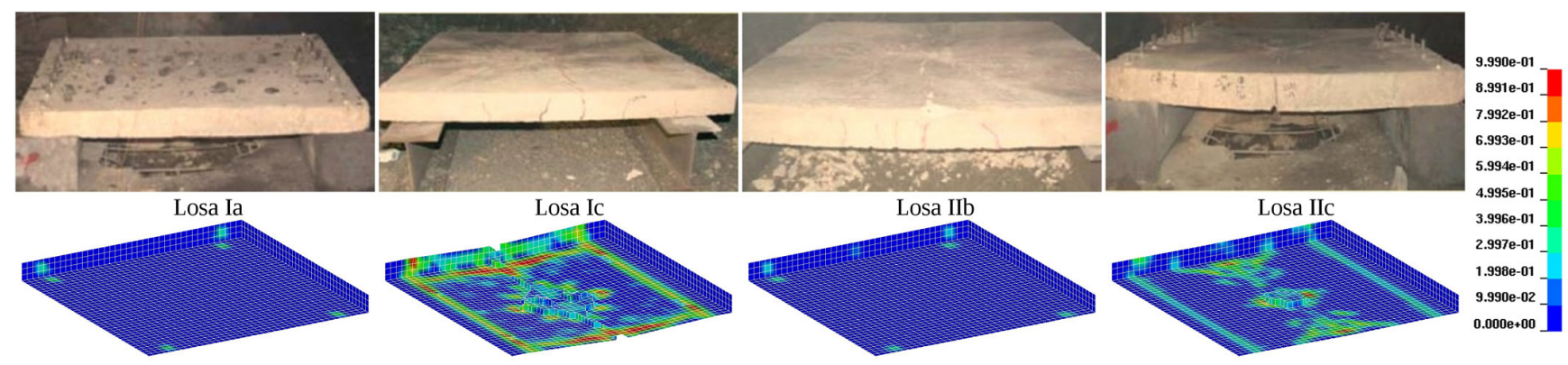

Figura 3. Modelado de los ensayos de Silva y Lu (11) mediante elementos de continuo 3D con LS-DYNA.

\subsection{Descripción de modelos de columna y losa}

Se analiza una columna simplemente apoyada de hormigón de $40 \mathrm{MPa}$ con doce barras corrugadas longitudinales de $20 \mathrm{~mm}$ de diámetro con cercos transversales de $8 \mathrm{~mm}$ de diámetro cada $30 \mathrm{~cm}$ de acero B500S. Las dimensiones de la columna son de $3 \mathrm{~m}$ de longitud y $45 \times 45 \mathrm{~cm}$ de sección.

El mallado para la columna consta de $6 \times 6 \times 40$ elementos sólidos 3D de hormigón modelados mediante material CSCM (características: $\rho=2320 \mathrm{~kg} / \mathrm{m}^{3}, f^{\prime} c=40 \mathrm{MPa}, D_{\text {agg }}=19 \mathrm{~mm}$, $\left.\xi_{\text {erode }}=1,05\right)$, con 16 barras modeladas mediante material piecewise linear pasticity (características: $\rho=7850 \mathrm{~kg} / \mathrm{m}^{3}$, $E=210 \mathrm{GPa}, v=0,3, \sigma_{v}=500 \mathrm{MPa}, \varepsilon_{u}=14 \%$ ) de diámetro $20 \mathrm{~mm}$ con 20 elementos cada barra representando el armado longitudinal, y 11 cercos de armado también modelados mediante material piecewise linear pasticity de diámetro $8 \mathrm{~mm}$ con 16 elementos cada cerco, cuatro por cada cara de la columna.

La losa reticulada (ver Figura 5) se construye mediante un forjado de casetones recuperables que crean huecos en la parte inferior de la misma. La armadura en este caso es compleja, con armado en los nervios, mallazo en la parte superior, etc. El modelo presentado usa hormigón de $30 \mathrm{MPa}$ y acero B50oS. Las dimensiones son de $8 \times 8 \mathrm{~m}$ de losa entre columnas con un canto total de $38 \mathrm{~cm}$.

El canto se divide en $30 \mathrm{~cm}$ de nervio más $8 \mathrm{~cm}$ de capa de compresión, excepto en los ábacos de las columnas en los que el canto es constante de $38 \mathrm{~cm}$ de hormigón. La sección se divide en casetones de $80 \times 80 \mathrm{~cm}$. Los nervios tienen un ancho de $12 \mathrm{~cm}$, quedando así un hueco de $68 \times 68 \mathrm{~cm}$ en cada casetón de $30 \mathrm{~cm}$ de profundidad. Los ábacos tienen $3,2 \times 3,2$ m de dimensión sobre una columna, de forma que, en un módulo, su planta es de 1,6 × 1,6 m. El armado, con recubrimiento de $2,5 \mathrm{~cm}$, consta de:

- En banda de soportes:

- En la cara superior, $2 \varnothing 16$ por nervio, $L=2 \times 2,65 \mathrm{~m}$.

- En la cara inferior, $1 \varnothing_{20}$ por nervio, $L=8,50 \mathrm{~m}$.

- En banda central:

- En la cara superior, $2 \varnothing 12$ por nervio, $L=2 \times 2,65 \mathrm{~m}$.

- En la cara inferior, $1 \varnothing_{16}$ por nervio, $L=8,50 \mathrm{~m}$.

- En cada ábaco:

- En la cara superior, $2 \varnothing_{16}$ entre nervios, $L=2 \times 1,60 \mathrm{~m}$.
-Armadura de punzonamiento, $4 \times 4 \varnothing 16$ con cercos $\varnothing 8$ a 0,20 .

- En la capa de compresión: mallazo de $\varnothing 8$ a o,10 m.

- Cercos en los nervios: $4 \varnothing 8$ a 20 m por nervio en banda de soportes.

El comportamiento de estos modelos se discute en el apartado siguiente, comparando sus resultados con los modelos de elementos estructurales.

\section{MODELOS DE ELEMENTOS FINITOS ESTRUCTURALES: VIGAS Y LÁMINAS}

\subsection{Características y limitaciones de los modelos de vigas y láminas}

En el modelo de elementos estructurales para la columna se usan elementos lámina cruzados en el eje de la viga con material de hormigón EC2 y vigas excéntricas para el armado longitudinal que se unen a los nodos en el cruce de las láminas, de forma que quedan unidas las doce barras al eje de la columna considerando su excentricidad. Las dimensiones de las láminas se ajustan para obtener una misma masa y una misma inercia seccional que en la columna real. Se emplean láminas para el hormigón en vez de vigas por que en LS-DYNA las láminas son capaces de recoger la presión de la onda explosiva, mientras que las vigas no. No es posible incluir el armado transversal de forma segregada, por lo que se tiene en cuenta como una cuantía de armadura en el modelo de hormigón EC2. En la Figura 4 se muestra las mallas y la validación.

Para la losa se usa el material EC2 para el hormigón y acero elastoplástico para las armaduras, con las mallas dispuestas de modo que la malla del hormigón y la malla del acero tengan nodos coincidentes. En las armaduras se emplea la opción OFFSET de LS-DYNA para tener en cuenta la excentricidad de las mismas con respecto al plano de la losa.

\subsection{Validación de la respuesta con elementos estructurales}

Entre los modelos de elementos de continuo y de elementos estructurales tanto para columna como para losa se realizan dos comprobaciones: en primer lugar una comparación cuasiestática, y en segundo lugar una comparación dinámica frente a acción explosiva.

En el caso de la columna se utiliza el modelo definido en el apartado 3.3. El modelo de elementos estructurales viga 

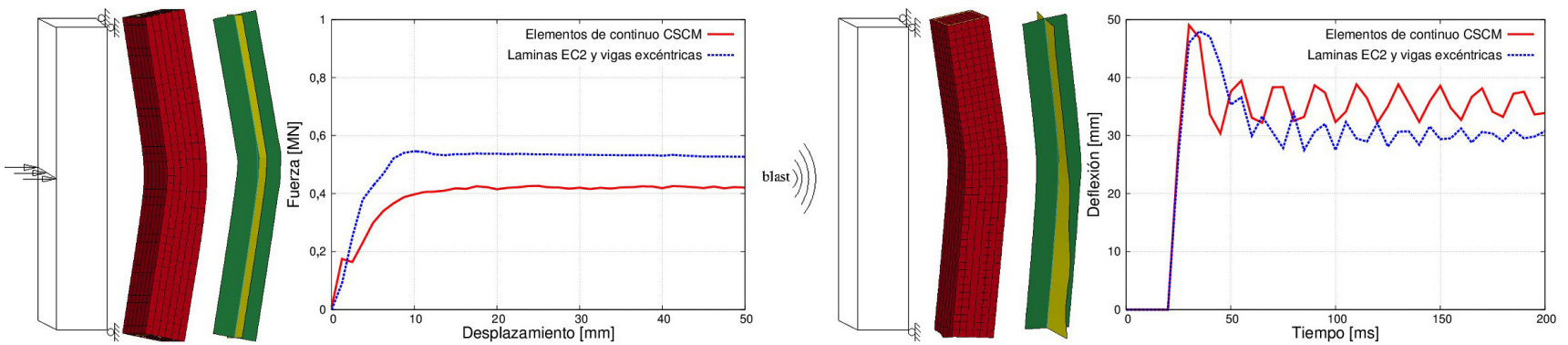

Figura 4. Comparación de modelos con elementos de continuo 3D y elementos estructurales. Evolución del desplazamiento en el centro de la columna -en las mallas deformadas se aplica un factor de amplificación de 5-a) Flexión cuasiestática. b) Respuesta dinámica para una carga de $400 \mathrm{~kg}$ de TNT a $4 \mathrm{~m}$ de distancia.

y lámina usa una configuración de dos láminas en cruz con material EC2 para hormigón y vigas para representar las armaduras. Para obtener la misma masa e inercia seccional que en la columna real se toman $30,7 \mathrm{~cm}$ de espesor con $45 \mathrm{~cm}$ de ancho de lámina y $\rho=685 \mathrm{~kg} / \mathrm{m}^{3}$. La malla es de $20 \times 4$ elementos lámina y 10 elementos viga por cada una de las barras de armado. Los materiales son hormigón EC2 (características: $\rho=685 \mathrm{~kg} / \mathrm{m}^{3}, f^{\prime} \mathrm{c}=40 \mathrm{MPa}, f^{\prime} t=4 \mathrm{MPa}$ ) y acero mediante material piecewise linear pasticity (características: $\rho=7850 \mathrm{~kg} / \mathrm{m}^{3}$, $\left.E=210 \mathrm{GPa}, \mathrm{v}=0,3, \sigma_{v}=500 \mathrm{MPa}, \varepsilon_{u}=14 \%\right)$.

La Figura 4a muestra la comparación de la respuesta de la columna a un movimiento transversal lento impuesto en su centro, que produce una flexión cuasi-estática en ella. Las curvas de ambos modelos tienen una buena correlación en cuanto a la historia de desplazamiento y los puntos de plastificación. El modelo de elementos estructurales es un poco más rígido debido a que en el otro modelo los elementos van plastificando progresivamente, mientras que en éste, al haber menos elementos en la sección, plastifican para una carga mayor. La Figura 4b muestra la deformación producida en el centro de la columna por una explosión de $400 \mathrm{~kg}$ de TNT a una distancia de $4 \mathrm{~m}$, aplicándose la acción explosiva en todas sus caras, observándose que también existe buena correlación.

Para la losa se usa un modelo de láminas EC2 para hormigón y vigas de armado excéntricas, con un mallado de $20 \times 20$ elementos lámina y armados equivalentes al modelo $3 \mathrm{D}$ cada dos elementos lámina. Los materiales son: hormigón EC2 (características: : $\rho=2300 \mathrm{~kg} / \mathrm{m}^{3}, f^{\prime} c=30 \mathrm{MPa}, f^{\prime} t=3 \mathrm{MPa}$ ). Acero mediante material piecewise linear plasticity (características: $\left.\rho=7850 \mathrm{~kg} / \mathrm{m}^{3}, E=210 \mathrm{GPa}, v=0,3, \sigma_{\mathrm{v}}=500 \mathrm{MPa}, \varepsilon_{u}=14 \%\right)$.

Este modelo consta de un número mucho menor de elementos que el modelo de continuo equivalente y con tamaños mayores de los mismos, lo que reduce considerablemente el coste computacional (Tabla 2). Las cuantías de armadura y su excentricidad son equivalentes en ambos, y como se muestra en la gráfica de la Figura 5 la respuesta a la flexión cuasi-estática, teniendo en cuenta la complejidad del modelo, es suficientemente aproximada en cuanto a desplazamiento y sucesivos procesos de plastificación.

Tabla 2. Comparación de tiempos de cálculo para los modelos de losa, con tiempo de simulación o,4 s.

\begin{tabular}{|l|c|c|}
\hline & $\mathbf{N}^{\mathbf{0}}$ de elementos & Tiempo cálculo \\
\hline Sólidos 3D & 18000o sólidos y 2900o vigas & $2 \mathrm{~h} 50 \mathrm{~min}(12 \mathrm{CPU}$ Xeon-X565) \\
\hline Láminas y vigas & 728 láminas y 916 vigas & $55 \mathrm{~s}$ (12CPU Xeon-X565) \\
\hline
\end{tabular}

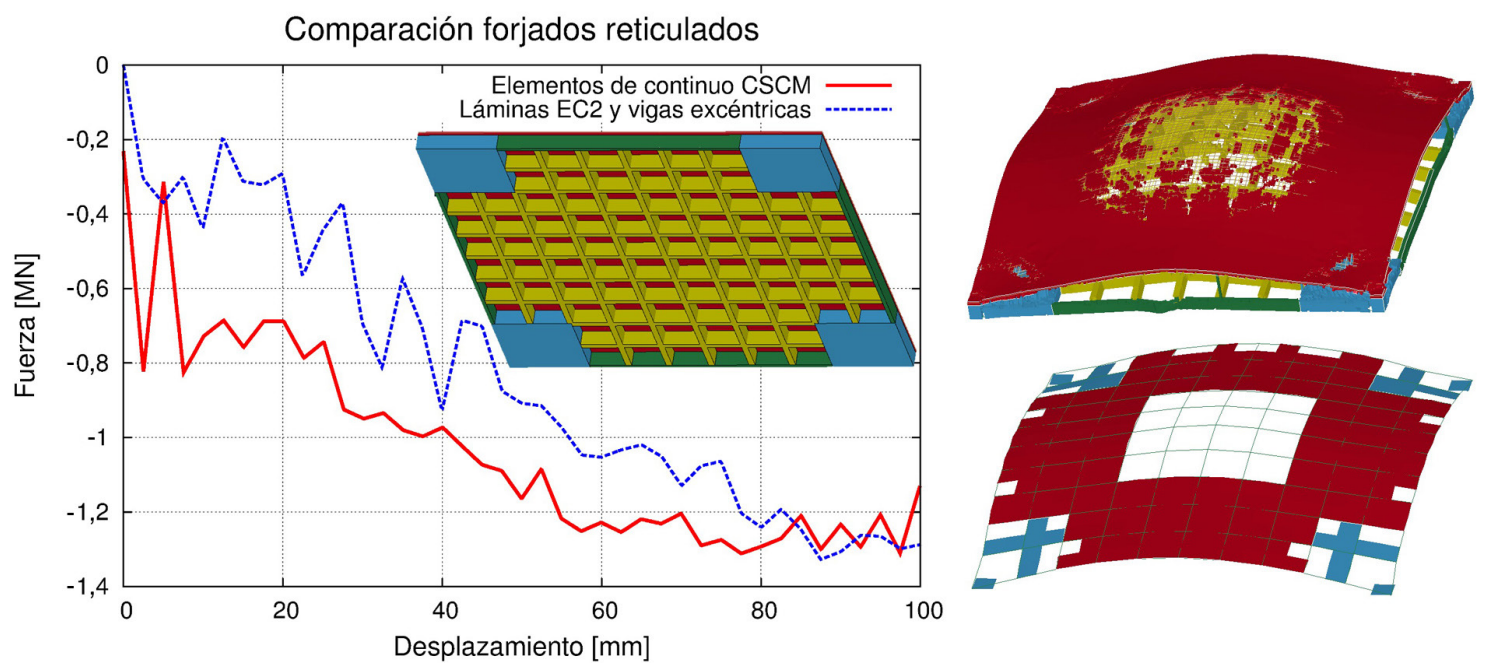

Figura 5. a) Comparación de la reacción frente a flexión de los dos modelos de losa reticulada mediante carga centrada.

b) Comparación: Malla deformada de modelo de elementos de continuo y modelo de elementos estructurales, sometida a $200 \mathrm{~kg}$ de TNT. 
La Figura 5 muestra también la comparación frente a una carga explosiva del modelo de elementos de continuo con el modelo de elementos estructurales cuando se somete a una carga centrada de $200 \mathrm{~kg}$ de TNT a dos metros de distancia bajo la losa. La acción explosiva aplica en todas las superficies de la cara inferior. En estos resultados el desplazamiento máximo, la tensión en las armaduras y las zonas erosionadas son similares. La conclusión es que el modelo de elementos lámina, que tiene un coste computacional mucho más bajo, es capaz de recoger en gran parte el comportamiento global de la losa completa.

Los resultados obtenidos permiten concluir que el modelado simplificado basado en elementos estructurales puede emplearse para evaluar edificios completos con estructura reticulada sometidos a acción explosiva.

\section{APLICACIONES: PÓRTICO AISLADO Y EDIFICIO COMPLETO}

\subsection{Pórtico aislado}

Se presenta en primer lugar una estructura completa simple, consistente en un pórtico compuesto por 4 columnas fijadas al suelo y una losa reticulada. Las columnas y losa empleadas son las referidas en los apartados anteriores. El tiempo de simulación es $0,2 \mathrm{~s}$ y la carga es de $200 \mathrm{~kg}$ de TNT a dos metros bajo la losa y centrada en el modelo iniciando la explosión en $\mathrm{t}=0,02 \mathrm{~s}$. La unión entre la losa y la columna se realiza mediante la unión de nodos y la superposición de armaduras. La Figura 6a muestra la deformación y rotura del pórtico comparando los modelos de elementos de continuo y los modelos de elementos estructurales de láminas y vigas.

En las gráficas de la Figura 6a se muestra la comparación de historias de velocidades entre ambos modelos, en puntos característicos de la estructura, como son el centro de la losa y la parte superior de las columnas: a la izquierda velocidades del extremo superior de la columna en las que se aprecia que la velocidad al inicio es negativa, pues la columna se desplaza hacia fuera por el efecto directo de la onda explosiva, pero rápidamente se vuelve positiva, debido al efecto de arrastre de la losa; a la derecha velocidad en el centro de la losa mostrando aumento brusco inicial de la velocidad cuando la onda explosiva llega a la losa, descendiendo rápidamente cuando la progresiva plastificación del resto de la estrutura limita su desplazamiento. Se observa una discrepancia en la parte final de la velocidad en el centro de la losa. Ésta es debida a que en el modelo de elementos estructurales la velocidad se mide en un único punto (el nodo central de la armadura) y en el modelo de elementos de continuo se mide como media de dos puntos (nodo central del mallazo superior y nodo central de la armadura inferior), y en este último modelo hay rotura local del mallazo que produce velocidades mayores.

El tiempo de cálculo es de 2 horas 17 minutos para el modelo de elementos de continuo y de 17 segundos para el de elementos estructurales, para un tiempo de simulación de 0,2 segundos, con 4 procesadores Xeon-X565.

\subsection{Edificio completo}

En segundo lugar (Figura 6b) se muestran los resultados que pueden obtenerse en el caso de un edificio de 3 plantas compuesto por $4 \times 4$ forjados reticulados en cada planta, modelado con elementos estructurales. Este modelo es capaz de estimar el daño causado en un edificio de estas características cuando se le somete a una carga explosiva en su interior. En este caso la carga es de $400 \mathrm{~kg}$ de TNT colocada en la primera planta del edificio a un metro de altura del suelo de la primera losa, que produce el daño observado en la figura. El tiempo de simulación está entre 0,1 y 0,5 segundos, suficiente para evaluar la acción de la explosión en la estructura. Con este tiempo de simulación y el modelo estructural simplificado el coste computacional es moderado ( $17 \mathrm{~min}$ con $12 \mathrm{CPU}$ Xeon-X565).

Las técnicas de modelado propuestas resultan adecuadas para la acción de explosiones fuertes en edificios completos, a pesar de lo cual existen algunas limitaciones a esta estrategia. Una de ellas es que no es posible evaluar directamente la presión que le llega por efecto de la explosión a una segunda losa cuando una primera ha roto y la onda de presión pasa a través de ella. La energía perdida por la onda explosiva en el proceso de rotura de la primera losa es difícil de estimar. Este fenómeno físico se puede evaluar mediante un modelo acoplado con interacción fluido-estructura (29) simulando la propagación de la onda explosiva en una malla de aire, pero sería necesario un número de elementos mucho mayor y de menor tamaño y una formulación más compleja, que conduce a un coste computacional excesivo. Otra de las limitaciones es que partes de la estructura pueden convertirse en proyectiles que impacten sobre partes posteriores del edificio. El parámetro de erosión empleado para simular el daño hace que se produzcan menos proyectiles en el modelo que en la realidad.

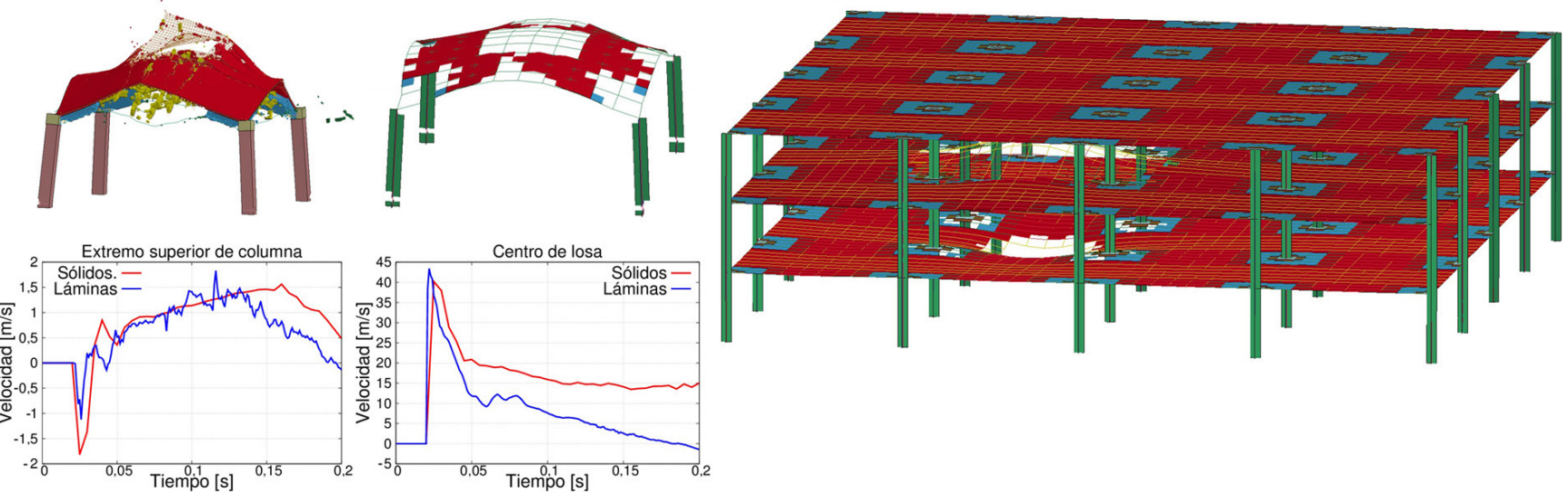

Figura 6. a) Explosión 200 kg de TNT centrada bajo pórtico a 2 m: Malla erosionada. Comparación de velocidades en el extremo superior de la columna y en el centro de la losa.b) Explosión de $400 \mathrm{~kg}$ TNT en el interior de un edificio. 
Este modelo puede ser usado también para estudiar el posible colapso progresivo, con el inconveniente de un mayor tiempo de cálculo, debido a que el tiempo de simulación tiene que aumentar, hasta valores de 3 a 10 segundos en este edificio de tres plantas, para poder evaluar los procesos de colapso y caída que se desarrollan en tiempo más largos que la explosión. Esto causa un incremento notorio en el tiempo de cálculo, pero éste es asumible.

\section{CONCLUSIONES}

Se presenta una metodología de modelado de escenarios de explosión sobre estructuras reticuladas de hormigón basada en modelos de elementos viga y lámina mediante los modelos de material EC2 y piecewise linear plasticity en LSDYNA, incluyendo la armadura sobre el hormigón de una forma realista. Estos modelos son calibrados con modelos detallados de elementos de continuo $3 \mathrm{D}$, con modelos de material CSCM y piecewise linear plasticity, mostrando una precisión suficiente. Se destacan a continuación las principales observaciones:

- Es necesario emplear modelos de elementos estructurales para el estudio de explosiones en edificios de varias plan- tas debido al coste computacional de los modelos detallados de elementos de continuo 3D.

- Los modelos de elementos estructurales, debidamente calibrados con modelos detallados de elementos de continuo $3 \mathrm{D}$, proporcionan una respuesta aproximada a la realidad.

- Existen limitaciones a esta metodología tales como la evaluación del efecto de la explosión en sucesivas plantas o la consideración del lanzamiento de proyectiles debido a la proyección de partes de la estructura, que requieren evaluación adicional para ser superadas.

- Esta metodología permite evaluar daños producidos por cargas explosivas y posibles mejoras de diseño frente a acción explosiva en estructuras completas reticuladas de hormigón.

\section{AGRADECIMIENTOS}

El trabajo aquí presentado se ha enmarcado en un proyecto de investigación en el que ha participado la Universidad Politécnica de Madrid, con el soporte de AENA, cuyo objetivo es modelar acciones explosivas sobre estructuras reticuladas de hormigón y mejorar la resistencia en el diseño de nuevas estructuras.

\section{REFERENCIAS}

(1) Crawford, J. E. (2002). Retrofit methods to mitigate progressive collapse. Glendale, CA: Karagozian \& Case Structural Engineers.

(2) Corres-Peiretti, H., Romero-Rey, E. (2008). Reconstrucción «Módulo D» aparcamiento Madrid Barajas T-4. En IV Congreso de Asociación Científico-técnica del Hormigón Estructural (ACHE). Valencia: ACHE.

(3) Wilkins, M. L. (1964). Calculation of elastic-plastic flow. En Alder, B. (Ed.) Methods in computational physics, vol. 3, pp. 211-263. New York: Academic Press.

(4) Wilkins, M., Blum, C. E., Grantham, P. (1975). A method for computer simulation of problems is solid mechanics and gas dynamics in three dimensions and time. Tech. rep. (rept. UCRL-51574). California CA (USA): Lawrence Livermore Laboratory.

(5) Hallquist, J. O. (1983). Theoretical manual for DYNA3D. Tech. rep. California CA (USA): Lawrence Livermore National Lab.

(6) Hallquist, J. O. (2006). LS-Dyna. Theory manual. http://www.lstc.com/pdf/ls-dyna_theory_manual_20o6.pdf.

(7) Pelessone, D. (1989). A modified formulation of the cap model. Tech. rep. (Gulf Atomics Report GAC19579). Defense Nuclear Agency under Contract DNA-001086-C-0277.

(8) Schwer, L. E., Murray, Y. D. (1994). A three-invariant smooth cap model with mixed hardening. International Journal for Numerical and Analytical Methods in Geomechanics, 18(10): 657-688, doi: http://dx.doi.org/10.1002/nag.1610181002.

(9) Simó, J., Ju, J. (1987). Strain and stress based continuum damage models. International Journal of Solids and Structures, 23(7): 821-840, doi: http://dx.doi.org/10.1016/0020-7683(87)90083-7.

(10) Magnusson, J. (2007). Structural Concrete Elements Subjected to Air Blast Loading (Ph.D. dissertation).

(11) Silva, P. F., Lu, B. (2009). Blast Resistance Capacity of Reinforced Concrete Slabs. Journal of Structural Engineering, 135(16): 708-716, doi: http://dx.doi.org/10.1061/(ASCE)ST.1943-541X.ooooo11.

(12) Morales-Alonso, G., Cendón, D., Gálvez, F., Erice, B., Sánchez-Gálvez, V. (2011). Blast Response Analysis of Reinforced Concrete Slabs: Experimental Procedure and Numerical Simulation. Journal of applied mechanics, 78(5), doi: http:// dx.doi.org/10.1115/1.4004278.

(13) Luccioni, B. M., Ambrosini, D., Danesi, R. (2003). Colapso estructural bajo cargas explosivas. Mecánica Computacional, XXII: 957-970.

(14) Luccioni, B., Ambrosini, R., Danesi, R. (2004). Analysis of building collapse under blast loads. Engineering Structures, 26(1): 63-71, doi: http://dx.doi.org/10.1016/j.engstruct.2003.08.011.

(15) Birnbaum, N., Cowler, M., Itoh, M., Katayama, M., Obata, H. (1987). AUTODYN-An interactive non-linear dynamic analysis program for microcomputer through supercomputers. En Transactions of 9 th Structural Mechanics in Reactor Technology, (pp. 401-406).

(16) Krauthammer, T., Cipolla, J. (2007). Building blast simulation and progressive collapse analysis. En NAFEMS (Ed.). http://www.nafems.org/downloads/public/buildingblast.pdf.

(17) Hibbitt, K. (2003). Abaqus/Explicit User's Manual. USA: Hibbitt, K, Sorenson Inc.

(18) Jayasooriya, R., Thambiratnam, D., Perera, N. J., Kosse, V. (2011). Blast and residual capacity analysis of reinforced concrete framed buildings. Engineering structures, 33(12): 3483-3495, doi: http://dx.doi.org/10.1016/j.engstruct.2011.07.011. 
(19) Akram, A.-O. (2008). Modeling and simulation of bogie impacts on contrete bridge rails using LS-Dyna. En 1oth International LS-Dyna Users Conference. Dearborn, Michigan USA.

(20) Goicolea, J. M. (2000, octubre). Estructuras sometidas a impacto. En Estructuras sometidas a acciones dinámicas (pp. 535-567). Barcelona: Centro Internacional de Métodos Numéricos en Ingeniería.

(21) Murray, Y. (2007). Users Manual For LS-DYNA Concrete Material Model 159. http://www.fhwa.dot.gov/publications/ research/infrastructure/pavements/05062/05062.pdf.

(22) Goicolea, J. M. (1992). Análisis termomecánico no lineal mediante métodos explícitos de diferencias finitas y elementos finitos. Revista Internacional de Métodos Numéricos para Cálculo y Diseño en Ingeniería, vol. 8,3: $235-265$.

(23) LSTC. (2013). LS-DYNA® Keyword User's Manual. http://www.lstc.com/download/manuals.

(24) Bermejo, M., Goicolea, J. M., Gabaldón, F., Santos, A. (2011). Impact and Explosive Loads on Concrete Buildings Using Shell and Beam Type Elements. En COMPDYN2011. 3rd ECCOMAS Thematic Conference on Computational Methods in Structural Dynamics and Earthquake Engineering. Corfu, Greece.

(25) Department of the Army. (1978). TM 5-855-1. Technical Manual. Fundamentals of protective design for conventional weapons. U.S. Department of the Army.

(26) Hyde, D. (1991). CONWEP, Conventional Weapons Effects Program. Vicksburg, Mississippi: US Army Engineers, Waterways Experiment Station.

(27) Randers-Pehrson, G., Bannister, K. A. (1997). Airblast Loading Model for DYNA2D and DYNA3D. Army Research Laboratory.

(28) Kingery, C., Bulmash, G. (1984). Air blast parameters from TNT spherical air burst and hemispherical surface burst. Ballistic Research Laboratories.

(29) Slavik, T. P. (2009). A Coupling of Empirical Explosive Blast Loads to ALE Air Domains in LS-DYNA. En 7th European LS-DYNA Conference. Stuttgart. 